\title{
Les tensions sur l'eau en Europe et dans le bassin méditerranéen Des crises de l'eau d'ici 2050
}

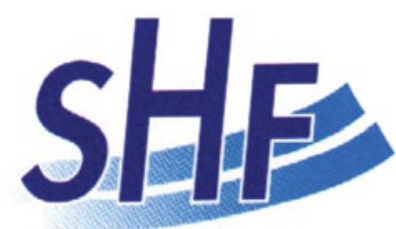

Société Hydrotechnique de France

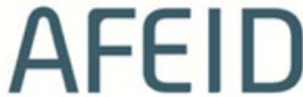

Association Française pour l'Eau, I'Irrigation et le Drainage
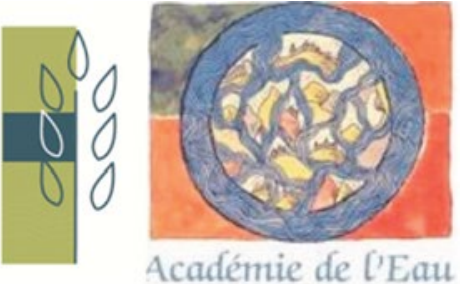

Jean Verdier (AFEID), Pierre-Louis Viollet (SHF)

Les auteurs remercient les membres du comité de pilotage du groupe inter-associatif : Laurent Bellet (EDF), Anna Dupont (SHF), Jeremy Gasc (ARTELIA), Ahmed Khaladi (CNR), Michel Lang (IRSTEA), Marc-Antoine Martin, Jean-Luc Redaud (Académie de l'Eau), Sara Fernandez (IRSTEA)

Ce document est une synthèse, aussi fidèle que possible, des conclusions ayant émergé des communications présentées lors d'un colloque inter-associatif tenu à Marne la Vallée du 7 au 9 octobre 2015. Les points de vue rapportés ici ne doivent donc pas être considérés dans leur intégralité comme un reflet fidèle des positions personnelles des auteurs ou des membres du comité de pilotage.

RÉSUMÉ. - Le changement climatique aura pour conséquences une réduction de la pluviométrie, particulièrement sur la zone méditerranéenne. Il y aura aussi une réduction de l'enneigement et des glaces en altitude et de nombreux cours d'eau alimentés par la fonte des neiges et des glaces subiront des réductions importantes de leurs régimes l'été, quand le besoin d'eau est justement plus grand. Le changement climatique se superpose à d'autres changements globaux , démographiques notamment. La surexploitation des nappes entraîne une baisse de leur niveau, et une dégradation de la qualité de l'eau. Des conséquences importantes sont attendues pour l'agriculture, la production d'électricité renouvelable hydraulique, la qualité de l'eau, les écosystèmes aquatiques et la biodiversité, le développement économique et l'exode rural. Des crises majeures sont attendues d'ici 2050. Entre les rives Nord et Sud de la Méditerranée, les évolutions présentent des différences, mais sont à bien des égards similaires, la situation étant bien plus grave sur la rive Sud. Des mesures d'adaptation existent : gestion de la demande, nouvelles pratiques agricoles, recyclage, construction de réservoirs et d'usines de dessalement mais aussi améliorations des rendements et de la gestion des systèmes de production, transport et distribution. Elles demandent pour être déployées des prises de décision politiques et des moyens économiques. L'adaptation de la gouvernance devrait être mise en œuvre, afin d'améliorer la capacité à anticiper les crises.

Mots-clés : Changement climatique, ressource en eau, adaptation

\section{Water Tensions in Europe and in the Mediterranean: water crisis by 2050?}

\begin{abstract}
Abstract. Climate change will induce a rainfall decrease, especially in the Mediterranean region. There will be also a reduction of snow cover and ice sheet in altitude, causing a runoff decrease on rivers fed by spring and summer snow and ice melting, at the time of freshwater peak demand. Climate change and other global changes (ie demography) combine their effects. Groundwater over-exploitation induces a lowering of the water table, and water quality degradation. Important consequences are expected for agriculture, hydro renewable energy generation, water quality, aquatic ecosystems and biodiversity, economy development, and population displacements. Major water crisis can be expected by 2050 or earlier. Between the Northern and Southern banks of the Mediterranean, there are differences, but the situation is by many aspects similar, of course much more severe on the South than the North. Adaptation measures do exist : demand management, new agricultural practices, water recycling, reservoir building and desalinisation plants as well as the improvement of efficiency and of production, transportation and distribution systems. The implementation of those solutions requires political decisions and economical inputs. Governance adaptation should be developed to improve the water crisis anticipation capacity.
\end{abstract}

Key-words: Climat change, water resources, adaptation 


\section{PRÉAMbule}

Depuis 2009, la SHF, l'AFEID, l'Académie de l'Eau et l'ASTEE ont mené des réflexions trans-disciplinaires et intersectorielles sur les approches prospectives de la gestion de l'eau dans un contexte de changements globaux.

Un premier séminaire avait été organisé à Paris les 25 et 26 mai 2011. Il a permis de faire une synthèse des représentations des principales tensions liées à l'eau à l'échelle mondiale, à partir de plusieurs études à caractère prospectif. Un second séminaire s'est tenu les 30 et 31 mai 2013, centré sur une perspective nationale française, replacée dans ses contextes européen et méditerranéen.

Un rapport de synthèse publié en janvier $2014^{1}$ indiquait que :

- le risque de crises de l'eau à l'horizon 2050 est bien avéré, en Europe et dans le bassin Méditerranéen,

- le facteur humain sera particulièrement important dans la capacité des sociétés modernes à affronter les tensions sur l'eau et à réaliser des choix,

- il sera nécessaire de mettre en place des politiques d'adaptation.

Un troisième colloque organisé par la SHF, l'AFEID et l'Académie de l'Eau, avec le soutien de l'Union des Ingénieurs et Scientifiques Francophones, s'est tenu du 7 au 9 octobre 2015 à l'Ecole des Ponts Paris Tech à Marne-la-Vallée. Il a permis d'approfondir les questions liées aux futures tensions sur la gestion de l'eau en Europe et dans le bassin méditerranéen d'ici à 2050. Le présent document est une synthèse des conclusions les plus marquantes de ce colloque.

Les ressources en eau douce sont mobilisées :

- pour l'approvisionnement en eau des populations humaines,

— pour la production agricole (alimentation, fibres, énergie, etc.),

- comme intrant de procédés industriels, notamment en tant que réfrigérant,

- pour la production d'énergie hydraulique (renouvelable et flexible),

— pour la navigation et la flottabilité,

- en tant que milieux des écosystèmes aquatiques,

- comme constitutives des patrimoines paysagers et culturels.

Ces différents usages peuvent être en compétition. Des oppositions entre usagers d'un même groupe peuvent également apparaître lorsque leurs demandes potentielles cumulées excédent les capacités de la ressource ou détériorent sa qualité (situation de rareté). De telles situations de conflits sont apparues dès la plus haute antiquité, donc indépendamment de tout changement climatique, et l'analyse de leurs modalités de règlement, des principes qui les ont inspirés, peuvent être source d'inspiration pour relaxer des tensions présentes ou pour prévenir des crises potentielles ${ }^{2}$. Certaines dispositions anciennes ayant résisté à l'épreuve du temps mériteraient une attention toute particulière, comme :

- l'institution de l'eau en tant que bien commun inaliénable dans le droit romain antique, disposition reprise dans le code de l'environnement français actuel et objet d'un intérêt croissant de la part de nombreux pays ;

1. Fernandez \& al., op. cit.

2. Ruf, op. cit.
- le tribunal des eaux de Valence (Espagne), attesté au $\mathrm{XIII}^{\mathrm{e}}$ siècle mais d'origine encore plus ancienne, et toujours actif de nos jours,

- la création, en 1291 par Philippe le Bel, de fonctionnaires d'État chargés de veiller à un bon usage des eaux et forêts, fonctionnaires dont le corps est toujours particulièrement actif dans la gestion nationale des ressources en eau.

Des crises contemporaines locales, indépendantes de changements globaux, peuvent également contribuer à une réflexion sur l'émergence de conflits liés à l'eau et à de possibles approches pour les dépasser.

La crise d'approvisionnement en eau de grandes villes algériennes, dont Alger, ayant culminé en 2001, en est un exemple exposé lors du colloque. On peut également citer les cas de Paris au XIX siècle ou, plus récemment, de Barcelone ${ }^{3}$.

Si le changement climatique induira rapidement des diminutions des ressources en eau dans le bassin méditerranéen, les évolutions démographiques auront également un fort impact sur la disponibilité de la ressource par habitant. Ainsi les pays des rives méridionale et orientale de la Méditerranée qui comptaient 235 millions d'habitants en 2000 devraient en avoir de l'ordre de 420 millions en 2050, alors que les ressources en eau de surface devraient diminuer de $30 \%$ à $40 \%$.

Des défauts de perception de réalités complexes peuvent aussi voir des crises où il n'y en a pas, voire induire ou accroître des crises de l'eau, comme cela a été présenté pour la Palestine ${ }^{4}$.

Enfin, il est essentiel de garder à l'esprit le caractère chaotique des évolutions de ressources en eau induites par le changement climatique, la baisse anticipée sur le pourtour méditerranéen n'étant qu'une tendance forte sur le long terme pouvant être masquée ou exagérée par des variations climatiques à court terme. La disponibilité de la ressource dépend en effet beaucoup de la pluviométrie et cette dernière peut varier fortement d'une année sur l'autre, notamment du fait de perturbations du cycle du front intertropical.

La présente synthèse ne concerne que les questions de pénuries et de partage de l'eau. La question des excès d'eau (inondations) liés au changement climatique n'est pas abordée ici.

\section{CONSTATS}

\section{II.1. Réduction de la pluviométrie.}

Il faut s'attendre à des évolutions dans la pluviométrie, dans le sens d'une diminution des pluies sur l'Europe du Sud et le monde Méditerranéen. Cette diminution pourra être dans l'ensemble de l'ordre de 20 à $50 \%$ d'ici 2080-2100, inégalement répartie entre les saisons et les régions ${ }^{5}$, et elle se manifeste déjà de façon importante au sud de la Méditerranée.

Au Maroc, par exemple, la pluviométrie moyenne interannuelle a déjà diminué de 580 à $400 \mathrm{~mm} / \mathrm{an}$ depuis 1980 ; ce pays voit d'ailleurs de grandes disparités géographiques, la pluie se concentre surtout dans le nord-ouest du pays (51\% des précipitations sur $7 \%$ du territoire) ${ }^{6}$.

Il y aura moins de chutes de neige en altitude.

\footnotetext{
3. Barraque, op. cit.

4. Fustec, op. cit.

5. Prudhomme, op. cit.

6. Sinan, op. cit.
} 


\section{Climate Change: Relative change in mean annual precipitation $1980 / 1999$ to $2080 / 2099$}

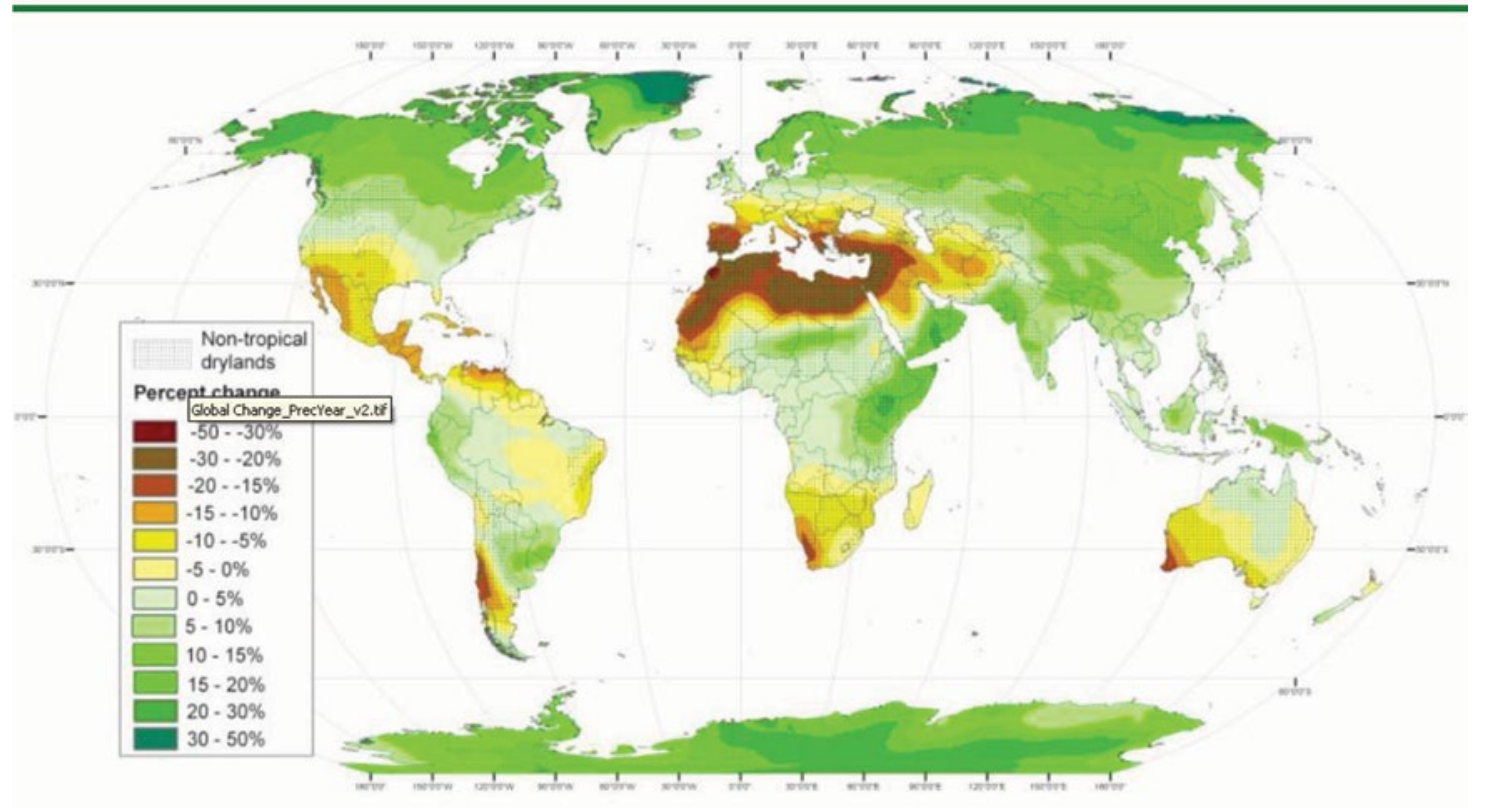

Relative change of mean annual precipitation $1980 / 1999$ to $2080 / 2099$, scenario A1b, average of 21 GCMs (compiled by GIS Unit ICARDA, based on partial maps in Christensen et al., 2007)

Figure extraite de Prudhomme, op. cit.

\section{II.2. Les nappes en surexploitation}

Conséquence directe de la diminution de la pluviométrie, l'alimentation des nappes d'eau souterraines renouvelables se trouve déjà réduite d'autant. Les prélèvements dans ces nappes étant par ailleurs en augmentation, les nappes se trouvent surexploitées et leur niveau baisse.

L'oasis de Marrakech, irriguée depuis l'antiquité par des galeries drainantes (qanats ou khettaras), exploitant par écoulement gravitaire la nappe alimentée par les pluies tombant sur l'Atlas, a vu le débit de ces galeries réduit d'un facteur 6 depuis 1975 . Le niveau de la nappe qui les alimente a baissé de $18 \mathrm{~m}$ en 24 ans $^{7}$.

Un autre constat est celui de l'exploitation de ressources en eau non renouvelables, nappes profondes ou nappes fossiles. Une fois épuisées, ces ressources le seront pour les générations à venir.

\section{II.3. Les débits naturels des cours d'eau diminuent, surtout l'été}

Les sens des évolutions des débits sur le pourtour méditerranéen ne laissent plus la place au doute, même si, en raison des incertitudes sur les modèles hydrologiques ${ }^{8}$, des incertitudes demeurent sur leurs quantifications.

Conséquence directe de la diminution des précipitations, les débits annuels des cours d'eau vont diminuer, Comme il y aura moins de neige et de glace en altitude, de nombreux cours d'eau évolueront d'un régime nival (alimentation par la fonte des neiges et de la glace au printemps et en été)

7. idem

8. Incertitudes sur le transfert pluie-débit, et sur l'évapotranspiration. à un régime pluvial (alimentation par les pluies). Ainsi, les débits moyens pourraient être plus élevés en hiver, mais surtout les réductions des débits seront particulièrement importants au printemps et en été (40 à $50 \%$ en France), qui sont les périodes sur lesquelles le besoin en eau agricole est le plus important.

Les conséquences du changement climatique auront aussi une influence sur la fréquence des extrêmes hydrologiques (hautes et basses eaux) en Europe du Sud et Méditerranée ; ces évolutions seront les suivantes":

- les périodes de basses eaux (étiages) seront plus fréquentes, toutes saisons confondues,

- les crues seront plus fréquentes en été,

tendances amplifiées sur la zone Méditerranéenne.

Prenons quelques exemples en zone tempérée : en 2050, le pic de débit annuel naturel (hors gestion des barrages) de la Seine à Paris, en mars-avril, pourrait être plus important de 20 à $30 \%$, tandis que le débit d'étiage d'été, d'août à octobre, pourrait être plus bas de 30 à $40 \%{ }^{10}$. Dans le canton de Vaud, en Suisse, les bassins hydrographiques du Jura et des Alpes verraient d'ici 2080 leurs débits réduits de 30 à $40 \%$ en été 11 .

\section{II.4. Le Sud de la Méditerranée en état de pénurie hydrique}

Les pays du Sud de la Méditerranée et du Proche Orient sont déjà en état de pénurie hydrique, ou bien vont

\footnotetext{
9. Prudhomme, op. cit.

10. Dorcies et al., op. cit. Voir aussi l'étude Explore 2070 (de Lacaze,

11. Milano \& al., op. cit.
} op. cit.) 
prochainement s'y trouver. Le seuil dit « de pénurie » de $500 \mathrm{~m}^{3} / \mathrm{habitant} / \mathrm{an}$ est déjà franchi en Algérie $\left(500 \mathrm{~m}^{3} / \mathrm{h} / \mathrm{a}\right)$, au Sahel tunisien $\left(415 \mathrm{~m}^{3} / \mathrm{h} / \mathrm{a}\right)$, en Lybie $\left(107 \mathrm{~m}^{3} / \mathrm{h} / \mathrm{a}\right)$. Le Maroc devrait passer en dessous de ce seuil vers 2020.

\section{DES MENACES ET DES CRISES}

\section{III.1. Des évolutions qui sont ou vont probablement être subies sous la pression des stress hydriques}

Conséquences directes des constats rappelés ci-dessus, des menaces planent sur un certain nombre d'activités humaines, de secteurs économiques, ainsi que sur la biodiversité. Ces menaces se traduisent déjà par des évolutions décidées implicitement sous la pression du manque d'eau.

\section{III.1.1. L'agriculture bénéficiera de moins d'eau}

Il y aura une réduction des volumes prélevables pour l'agriculture. que ce soit par prélèvements sur les cours d'eau ou par pompage dans les nappes. A noter que ces prélèvements résultent souvent de "droits d'eau " anciens, ce qui peut rendre particulièrement confuse et difficile à mettre en œuvre une politique rationnelle de réduction des prélèvements.

En Espagne, par exemple, les forages réalisés avant 1985 sont considérés comme relevant du domaine privé, ce qui $n^{\prime} e s t$ plus le cas de ceux réalisés après cette date ${ }^{12}$.

\section{III.1.2. Un développement industriel freiné dans les pays du Sud.}

Le manque d'eau pourrait entraîner une plus grande difficulté, voire une impossibilité, de développer des activités industrielles dans des régions actuellement peu développées et qui souhaiteraient se doter d'activités industrielles, notamment en Afrique du nord.

Se pose aussi bien sûr la question des modèles de développement : les pays du Sud devront-ils innover pour ne pas suivre les modèles industriels développés dans le Nord, sur-consommateurs en eu et en énergie ?

\section{III.1.3. L'hydroélectricité : une énergie renouvelable menacée}

La réduction des débits et la pression des autres usages sur les quantités d'eau stockées dans les réservoirs entraîne une menace sur la production hydroélectrique, production qui représente une contribution importante aux énergies renouvelables, et qui constitue par ailleurs un moyen de production flexible pour suivre les pointes de consommation et pallier aux défaillances des autres énergies.

Le Maroc a déjà vu une réduction considérable de sa production hydroélectrique depuis $1970^{13}$. Sur le Rhône, il y a une perspective de réduction de 10 à $20 \%$ de la production hydroélectrique d'ici 2050 ; cette réduction devrait être concentrée entre avril et novembre ${ }^{14}$. Sur la Durance, la contribution de l'hydroélectricité à la flexibilité de la production sera réduite $e^{15}$.

\footnotetext{
12. Cabezas \& Cabrera, Herera, op. cit.

13. Hasnaoui, op. cit.

14. Graff \& al. op. cit.

15. Sauquet \& al., op. cit.
}

\section{III.1.4. L'exode rural et les mouvements migratoires vont s'amplifier}

Dans les régions en situation de pénurie, des paysans abandonnent déjà leurs terres, et ce phénomène peut être cause de flux migratoires importants.

Cette situation se présente déjà, par exemple dans le Sahel tunisien : des paysans abandonnent les terres qu'ils ne peuvent plus cultiver pour se diriger vers les zones touristiques, qu'ils espèrent constituer pour eux des bassins d'emplois ${ }^{16}$.

\section{III.1.5. La qualité des eaux et la biodiversité vont être dégradées}

La surexploitation des nappes s'accompagne déjà dans certains pays comme par exemple le Maroc et l'Algérie d'une réduction de la qualité de leur eau ${ }^{17}$.

Par ailleurs, dans les cours d'eau, les basses eaux d'été devenant plus sévères et plus fréquentes, la qualité des habitats aquatiques sera dégradée, et le respect des débits réservés sera plus difficile.

\section{III.1.6. Les échanges transfrontaliers deviennent plus durs}

Dans une situation croissante de tension entre la demande et la ressource en eau, la question des transferts d'eau transfrontaliers, en premier lieu par les fleuves transfrontaliers, va se durcir. L'eau devient une source de pressions politiques, une denrée susceptible de se vendre.

La Turquie, par exemple, contrôle par ses grands barrages les eaux du Tigre et de l'Euphrate. Elle entend développer économiquement le sud-est de l'Anatolie, pour freiner les migrations, et jouer la carte du développement pour apaiser des tensions politiques (Kurdistan). Elle dispose dans ces réservoirs d'une capacité de stockage pluriannuelle, pourrait utiliser l'arme de l'eau pour faire pression sur les pays situés en aval, et envisage aussi d'exporter cette eau vers d'autres pays.

\section{III.2. Des crises : des décisions dans la douleur}

Les déclenchements de crise sont souvent multifactoriels et résultent généralement d'une combinaison de facteurs physiques, démographiques, économiques et sociaux. La combinaison entre ces diverses composantes est complexe et ses possibles trajectoires d'évolution difficiles à prédire précisément. De plus, le seuil de tolérance des tensions est difficile à estimer parce qu'éminemment variable au sein d'une même société. Il est donc difficile d'anticiper l'imminence d'une rupture majeure et d'événements violents.

Les moyens d'éviter des crises majeures peuvent impliquer des changements radicaux ou la mobilisation de moyens importants, il est donc très tentant d'en imaginer l'échéance lointaine et de surseoir à la mise en place de solutions pourtant considérées comme inéluctables. Il est donc fréquent que ce soit l'occurrence d'une situation intenable qui pousse à agir rapidement avec des solutions de court terme alors que des décisions prises dans un contexte moins conflictuel auraient pu être mieux réfléchies et avoir une meilleure efficacité à long terme.

Ainsi, en Algérie, il a fallu attendre qu'en 2001, il n'y ait plus de distribution d'eau potable que deux jours par

16. Gafsi, op. cit.

17. Haouchine \& al., op. cit. 
semaine dans la capitale pour que soit décidé de construire un ensemble d'usines de dessalement d'eau de mer susceptible d'augmenter notablement l'offre ${ }^{18}$.

\section{MESURES D'ADAPTATION}

Adaptation technique, économique, sociale, culturelle aux crises ou au risque avéré de crises de l'eau.

\section{IV.1. Techniques}

Presque toutes les mesures d'adaptation techniques ont un coût : investissement, exploitation et maintenance. Leur mise en œuvre sous-entend un consentement à payer de la part des utilisateurs, ce qui ne va pas forcément de soi lorsque l'eau a été longtemps considérée comme "gratuite ». Par ailleurs, plusieurs de ces solutions font l'objet de controverses, et chaque mesure n'est pas forcément pertinente partout.

Les solutions techniques évoquées ci-dessous doivent se cumuler avec les mesures de lutte contre le gaspillage de l'eau: fuites des réseaux, surconsommations agricoles, urbaines et des procédés industriels.

Le traitement des eaux usées est maintenant déployé dans de nombreuses villes. Il est partie prenante de presque tous les plans d'adaptation. Dans plusieurs villes, le recyclage des eaux usées est maintenant mis en place.

Windhoek, capitale de la Namibie, parvient à satisfaire le quart des besoins de la ville grâce à la réutilisation de l'eau. Alors que la demande en eau (liée à la croissance de la population) est passée de 6 à $27 \mathrm{Mm}^{3}$ entre 1967 et 2012, elle a mis en place, dès 1970, deux boucles de réutilisation des eaux usées. L'une dédiée à l'arrosage des espaces verts à partir d'eaux usées traitées, et la seconde à l'alimentation en eau potable à partir d'eaux usées (et eaux de surface) traitées. Le surplus des années humides est alors injecté dans l'aquifère situé sous une partie de la ville, ce qui permet d'assurer une réserve pour les années sèches ${ }^{19}$.

\section{IV.1.1. Le dessalement d'eau de mer}

Le coût du traitement a beaucoup diminué, et se situe actuellement aux alentours de $0,5 \mathrm{US} \$ / \mathrm{m}^{3}$. L'énergie électrique nécessaire peut être apportée par de l'énergie solaire, par des sources conventionnelles, ou des petits réacteurs nucléaires dérivés des réacteurs utilisée pour la propulsion nautique et fonctionnant en régime continu. La question de l'impact environnemental des rejets de saumure devrait sans doute cependant être mieux étudiée.

L'Algérie a commencé de déployer à grande échelle des centrales de dessalement après la crise hydrique de 2001 ; elle est maintenant dotée d'une trentaine de stations de dessalement, totalisant une capacité de plus de 2 millions de $\mathrm{m}^{3} / \mathrm{j} ; 12$ de ces stations ont une capacité comprise entre 100000 et $500000 \mathrm{~m}^{3} / \mathrm{j}^{20}$. Au Maroc cette option est à l'étude pour alimenter les plaines côtières, mais elle n'est encore pas intéressante d'un point de vue technico-économique sauf quelques cas particuliers : sa première station, à Agadir, utilise de l'énergie solaire, et fait l'objet d'un partenariat public-privé, garantissant sa pérennité économique ${ }^{21}$.

\section{IV.1.2. Adapter l'agriculture}

Il semble souhaitable de développer des pratiques agricoles plus économes en eau, qui permettent en même temps de maintenir l'emploi local. Par ailleurs, compte-tenu de la grande variabilité de la ressource d'une année sur l'autre, il est possible de développer des capacités d'adapter les choix agricoles de l'année à venir en fonction de la pluviométrie observée.

\section{IV.1.3. Développer les réservoirs}

L'intérêt des barrages-réservoirs est de permettre le soutien des étiages grâce à l'eau stockée en période de hautes eaux. Les plus grands réservoirs permettent par ailleurs de lisser la variabilité inter-annuelle de la ressource. La sédimentation dans les lacs de barrage, si elle n'est pas contrôlée, réduit cependant peu à peu la capacité utile des réservoirs.

L'Ebre, par exemple, voit son débit régularisé grâce aux barrages réservoirs existant sur son cours supérieur ${ }^{22}$. Au Maroc, il y a 139 barrages importants ; ce pays envisage de nouveaux schémas de barrages équipés d'amont en aval, pour réduire les menaces de sédimentation ${ }^{23}$.

Le stockage est donc une solution indispensable à l'adaptation mais elle ne peut pas être l'unique solution.

Par exemple, en 2050, pour compenser le déficit hydrologique des 844 bassins versants français, il faudrait plusieurs milliards de $m^{3}$ de stockage.

\section{IV.1.4. Développer des systèmes de gestion intelligents pour les barrages-réservoirs}

Les barrages réservoirs existant vont être pris entre de multiples contraintes: soutenir les étiages pour maintenir certains usages en aval, et maintenir la qualité de l'habitat aquatique, produire de l'électricité, écrêter les crues... L'arbitrage optimal entre ces contraintes demandera des systèmes d'exploitation intelligents, qui devront être alimentés par des réseaux plus denses de mesures hydrologiques, ainsi que par des modèles de prévision à court terme..

L'Ain, par exemple, est une rivière équipée en son cours amont du barrage-réservoir de Vouglans. Outre la production hydroélectrique, son eau est utilisée pour des usages agricoles dans la basse vallée, des activités récréatives, et son cours supérieur comme sa basse vallée constituent des habitats piscicoles importants et variés. En juillet 2015, une crise a pu être prévue et anticipée grâce à un système de prévisions et de modélisation avancés, en effectuant une "éclusée écologique » à partir du barrage amont ${ }^{24}$.

\section{IV.2. Gouvernance}

Dès lors que l'eau est considérée comme un bien commun, la définition des orientations de sa gouvernance impose de sortir du strict domaine de l'eau et d'immerger des questions proprement hydrauliques dans une vision politique plus globale incluant la production et la sécurité alimentaires, la production énergétique et la préservation des milieux.

L'ampleur des évolutions globales autour de la Méditerranée imposera des modifications importantes dans les modes de gestion de l'eau, pouvant se traduire par d'importants changements institutionnels ainsi que par des

18. Kettab, communication orale lors du colloque d'octobre 2015

19. Lafforgue, op. cit.

20. Haouchine, op. cit. et communication personnelle

21. Hasnaoui, op. cit. 
modifications profondes des relations entre les différents porteurs d'enjeux.

Pour que des modifications radicales puissent être acceptées et donner leur plein effet, il faut qu'elles soient porteuses d'un sens bien perçu et largement accepté par toutes les parties concernées. Si ce sens est nouveau (gestion de la demande, adaptation de l'agriculture, procédures d'exception en période de crise, etc.), cette acceptation requerra un processus de compréhension et d'appropriation nécessitant du temps. Ce processus nécessitera (i) une éducation à la compréhension des problèmes et des contraintes, (ii) un développement des capacités des acteurs engagés dans la gestion ou l'utilisation de la ressource pour imaginer d'autres modes de vivre ensemble en partageant un bien commun et (iii) une communication appropriée avec une bonne perception de toutes les facettes des problèmes.

L'ampleur du processus de mise en place de gouvernances susceptibles d'éviter durablement des conflits majeurs liés à l'eau dans le bassin méditerranéen impose d'anticiper les crises en gestation en dépit des difficultés qu'il y a à le faire, et qui ont évoquées plus haut.

\section{RÉFÉRENCES}

\section{Précédente synthèse sur les Tensions sur l'Eau :}

Sara Fernandez, Marc-Antoine Martin, Billy Troy, Jean Verdier, Pierre-Louis Viollet, Prospective et tensions sur l'eau. Des crises de l'eau en 2050? Note de synthèse, Janvier 2014, Publié par SHF, AFEID et Académie de l'Eau, Janvier 2014. http://www.shf-hydro.org/124-synthese prospectives_et tensions_sur_1_eau-7.html; http://www.academie-eau.org/fr/conferences-6.html

Documents de référence de trois exercices de prospective réalisées sur le périmètre France

\section{- Aqua 2030}

Nathalie Etahiri, Eau, milieux aquatiques et territoires durables 2030. Études \& documents, $n^{\circ}$ 91, Août 2013, Commissariat Général au Développement Durable. http://www.developpement-durable.gouv.fr/IMG/pdf/ED91.pdf

\section{- Explore 2070}

Xavier de Lacaze, Synthèse du projet Explore 2070, Intégration, Analyse systémique et stratégies d'adaptation. Ministère de l'Ecologie, du Développement Durable et de l'Energie, 2013. http://www.developpement-durable.gouv.fr/ Evaluation-des-strategies-d.html

\section{- Garonne 2050}

Françoise Carpy-Goular, La Garonne du futur, les futurs de la Garonne. Présentation au séminaire Prospectives et Tensions sur l'eau, 30 mai 2013.

Agence de l'eau Adour-Garonne.

Quelques communications au colloque "Tensions sur l'Eau en Europe et dans le bassin Méditerranéen, Oct 2015 », organisé par SHF, AFEID, Académie de l'Eau, UISF, publié par SHF,
25 rue des Favorites, 75015 Paris. Visuels sur http://www.shf-hydro. org/177-1-les_tensions_sur_1_eau_en_europe_et_dans_le_bassin mediterraneen-71.html

Bernard Barraque, EAU\& $3^{E}$, European Urban Water Crisis: The management dimension.

F. Cabezas, E. Cabrera, I. Morell, The water maze of southeast spain

D. Dorchies, G. Thirel, C. Perrin, J.-C. Bader, R. Thépot, Jean-Louis Rizzoli, C. Jost, S. Demerliac Climate change impact on water resources and reservoir management in the Seine River basin (France)

J. Fabre, D. Ruelland, A. Dezetter, B. Grouillet, Reducing the gap between water demand and availability under climate and water use changes: assessing the effectiveness and robustness of adaptation.

Klervi Fustec, From domestic water to agricultural water: how do donors contribute to the water crises in the occupied palestinian territories?

Fadia Gafsi, Analyse rétrospective de la tension sur l'eau dans le Sahel Tunisien

Benjamin Graff, Christophe Moretton, Sabrina Celié, Impact du changement climatique sur la production hydroélectrique du Rhône

Abdelhamid Haouchine, Fatima Zohra. Bouchareb-Haouchine, Brahiachid Chibane \& R. Nedjai, Assessment of risks related to coastal aquifers management in Algeria

Moulay Driss Hasnaoui, Quelle Stratégie de Développement Durable des Ressources en Eau pour le Maroc au-delà de 2030?

Africa de la Hera, Juan Maria Fornes, Emilio Custodio, Manuel Ramon Llomas, Andrès Sahuguilla, Water crisis in Spain since 1950: past and present, challenges and opportunities

Michel Lafforgue, Supplying water to a water stressed city:lessons from Windhoek

Ludovic Lhuissier, Veronique Lamblin, Eric Sauquet, Yannick Arama, Françoise Goulard, Pierre Strosser, Garonne 2050, A prospective Study

M. Milano, E. Reynard, N. Köplin, R. Weingartner, In light of seasonal climatic and anthropogenic changes, is the Vaud canton (Switzerland) vulnerable to water stress by the medium-term?

Alain Poirel, David Cheminet, Bruno Landru, Vincent Wawrzyniak, Marie Noëlle FAYE.... The passage of summer crises in the Ain River (France) : contributions of forecasting and lessons learned from the period 1994-2014

Christel Prudhomme, Impact of climate change on river flow s: global and European assessment for the 2080s time horizon

Thierry Ruf, Tensions climatiques, tensions politiques dans l'histoire contemporaine de l'eau au Nord et au Sud de la Méditerranée : une approche en termes de cycles institutionnels

Mohamed Sinan, Abdelaziz Belhouji, Impact of Climate Change On Water Resources in Morocco in the years 2020, 2050 and 2080 And Adaptation Measures

Eric Sauquet \& al., Water allocation and uses in the Durance River basin in the 2050s - Towards new management rules for the main reservoirs? 


\section{L'Etablissement public Loire}

au service du bassin

- Auvergne - Bourgogne - Centre-Val de Loire Languedoc-Roussillon • Limousin • Pays de la Loire • Rhône-Alpes Departements • Allier - Ardèche • Cher • Creuse Indre-et-Loire •
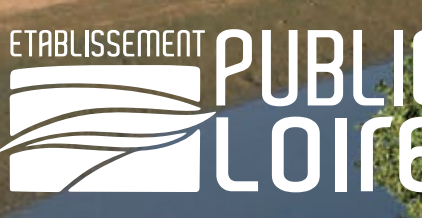

\section{GESTION AUTOMATIQUE DES CRUES ENTRANT AU BARRAGE DE VILLEREST : RETOUR D'EXPERIENCE}

Situé sur la Loire, en amont de la ville de Roanne, le barrage de Villerest est la propriété de l'Etablissement public Loire, également responsable de sa gestion.

L'ouvrage assure une double fonction : le soutien d'étiage en période de basses eaux et l'écrêtement des crues. Par son action sur les crues de la Loire amont, le barrage contribue significativement à leur réduction en Loire moyenne, tout en apportant une importante protection en Loire bourguignonne, comme cela a pu être observé lors des crues de 2003 et 2008.

Le barrage de Villerest est muni d'un calculateur qui comprend un système d'automates capable de piloter l'ouverture des vannes et de donner les consignes de débits à l'usine, dans le respect des dispositions du règlement d'eau, en situation normale (gestion à cote constante) comme en situation de risque de crue (débit entrant inférieur à $1.000 \mathrm{~m}^{3}$ ).

Un certain nombre de dysfonctionnements a été identifié problème de calcul du débit entrant après un déstockage (remise en automatique difficile), risque de dépassement de la cote maximale ; divergence entre les débits sortants calculés et les débits mesurés par jaugeage... C'est dans ce contexte qu'une démarche de modernisation des programmes du calculateur a été menée par l'Etablissement public Loire, avec le soutien financier de l'Europe (FEDER).

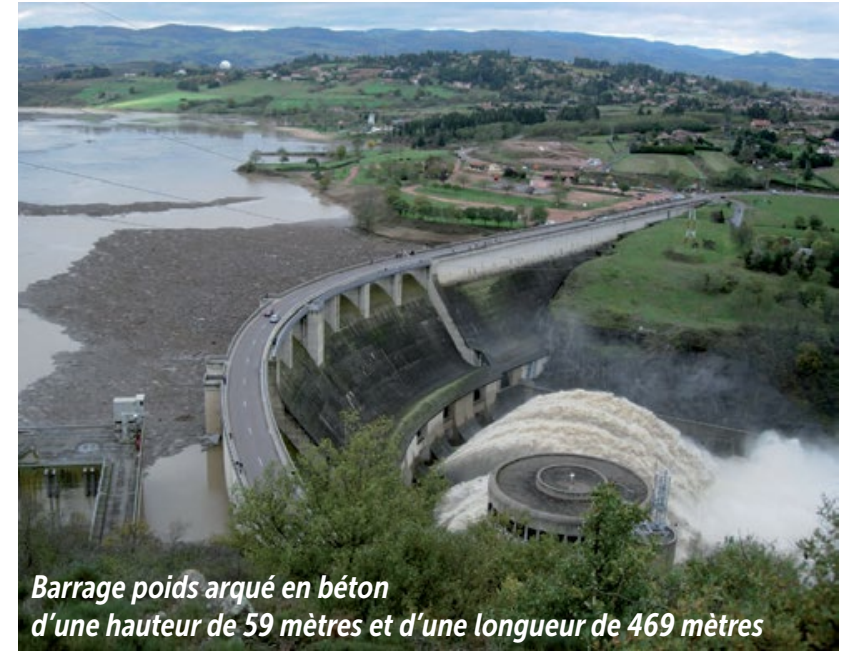




\section{UNE MÉTHOLOGIE APPLIQUÉE}

Dans l'ancien calculateur du barrage, le calcul du débit entrant ne suivait pas forcément la réalité hydraulique de l'ouvrage. En effet, un seul type de calcul était fait à partir de la variation de cote et du débit moyen sortant sur un pas de temps fixe. Or, selon si l'ouvrage est en configuration étiage ou en crue, la réactivité du plan d'eau n'est pas la même.

Dans le nouveau programme, le débit entrant est donc évalué à partir de la variation de cote et du débit moyen sortant sur des pas de temps variables. Pour les faibles débits, les pas de temps importants ont été privilégiés pour lisser les variations effectives du débit entrant et réduire les instabilités liées aux incertitudes sur les mesures de la cote. Le pas de temps a été progressivement réduit à mesure que le débit augmente pour pouvoir tenir compte des variations liées à un " coup d'eau ". Ainsi, le débit entrant de référence correspond au débit entrant moyen sur $24 \mathrm{~h}$ pour des débits inférieurs à $120 \mathrm{~m}^{3} / \mathrm{s}$, et à une moyenne sur $4 \mathrm{~h}$ pour un débit supérieurs à $300 \mathrm{~m}^{3} / \mathrm{s}$. Dans tous les cas, le débit entrant moyen est calculé au minimum sur $4 \mathrm{~h}$.

\section{DES RÉSULTATS PROBANTS POUR UNE GESTION DE RISQUE DE CRUE}

Le nouveau calculateur a été utilisé en mode opérationnel, notamment lors des risques de crues de janvier et de novembre 2014.

\section{- Evènement du 19 janvier 2014}

Le plan d'eau était initialement à la cote 314.75 m NGF. Le débit sortant était de $87 \mathrm{~m}^{3} / \mathrm{s}$.

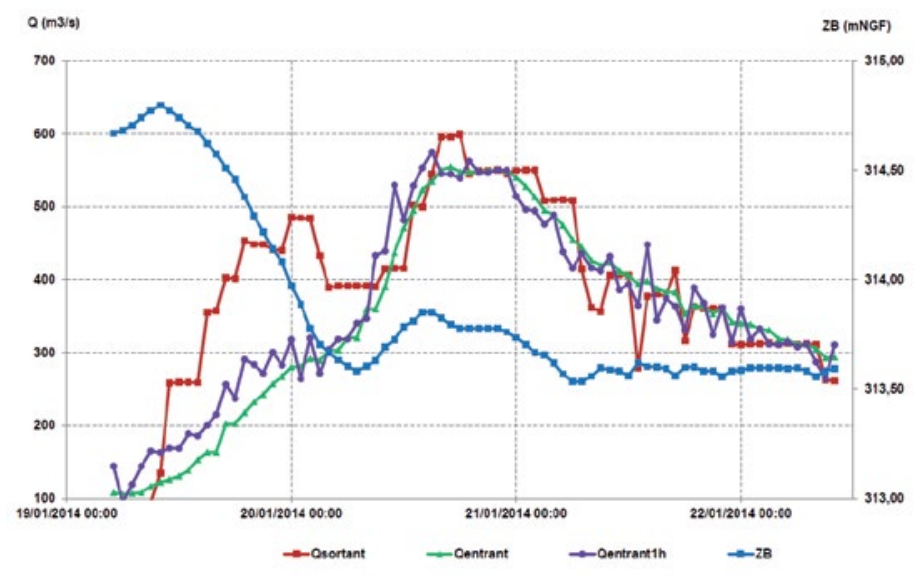

Fig.1 : Débits et cotes au barrage de Villerest lors de l'évènement de janvier 2014.

Constat: Conformément au règlement d'eau, durant cette période, la gestion au barrage a consisté à un déstockage à la cote 314 m NGF puis à un maintien de la cote à un niveau constant. L'abaissement de la cote a été fait à raison de 7 à $10 \mathrm{~cm}$ par heure. Durant tout l'évènement, le calculateur a parfaitement géré la phase de déstockage et le maintien de cote qui s'en suivit.
- Evènement du 12 octobre 2014

Le plan d'eau était initialement à la cote 303.45 m NGF. Le débit sortant initial était de $11.7 \mathrm{~m}^{3} / \mathrm{s}$. Suite à de fortes pluies cévenoles, un risque de crue s'est avéré.

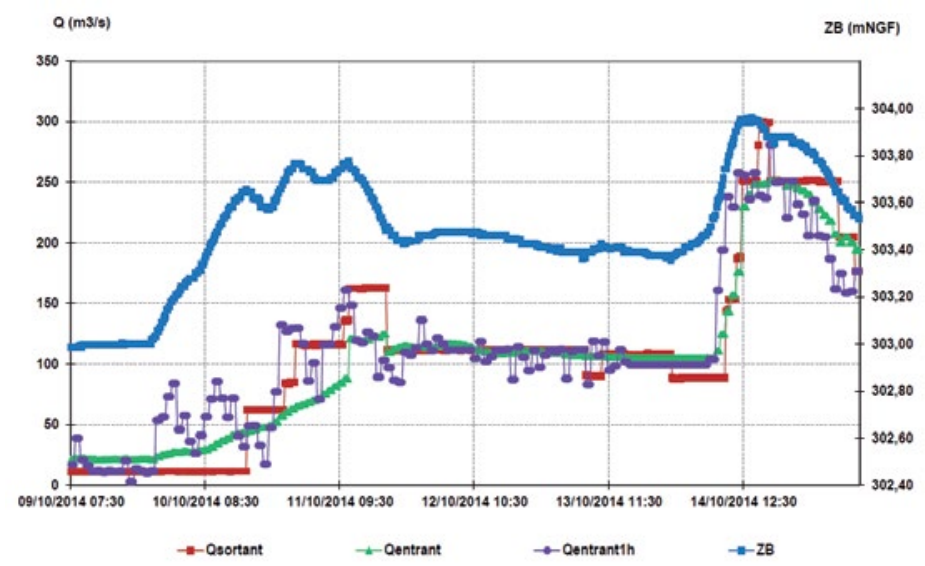

Fig.2: Débits et cotes au barrage de Villerest lors de l'évènement d'octobre 2014

Constat: Conformément au règlement d'eau, durant cette période, la gestion au barrage a consisté à maintenir la cote à un niveau constant, avec une cote maximale à ne pas dépasser de 304 m NGF. Compte-tenu de l'augmentation du débit entrant, l'atteinte de la cote 303.95 m NGF a entrainé la commande par le calculateur de l'ouverture d'un cran supplémentaire de $50 \mathrm{~m}^{3} / \mathrm{s}$ sur une vanne de demi-fond du barrage. Ce comportement est entièrement conforme aux algorithmes.

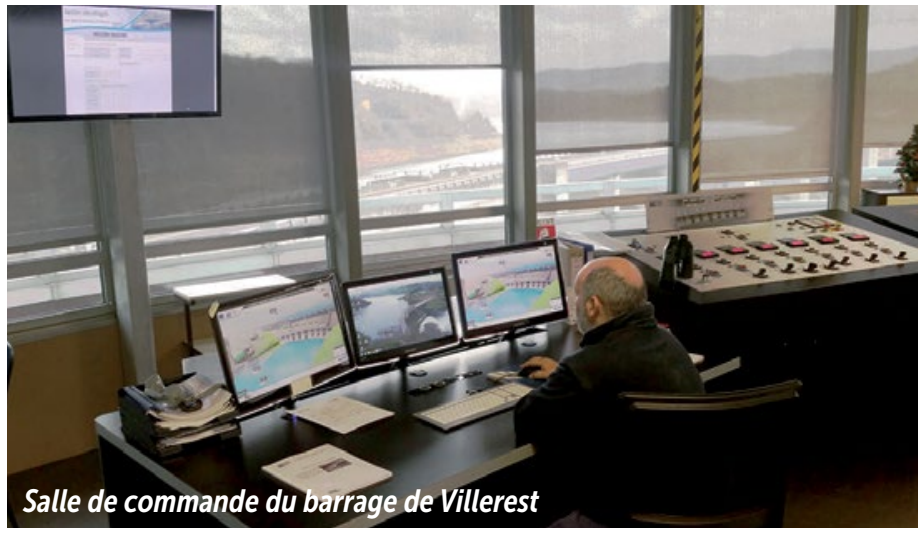

\section{UNE AUTOMATISATION EFFICACE MAIS N'ÉCARTANT PAS L'INTERVENTION HUMAINE}

La nouvelle méthode de calcul du débit entrant permet de paramétrer un déstockage moins violent que ce qui était prévu par l'ancien algorithme, et laisse à l'utilisateur la possibilité de choisir d'autres débits objectifs (exemple : Qe $+50 / 100 / 200 \mathrm{~m}^{3} / \mathrm{s}$ ).

La gestion des risques de crue durant l'année 2014 par les nouveaux algorithmes du calculateur s'est révélée plus efficace que dans la version antérieure, que ce soit en déstockage ou en maintien de cote. Néanmoins, pour garantir le respect du règlement d'eau, un contrôle systématique du comportement du calculateur est effectué par l'exploitant du barrage. 